\title{
EXPERIMENTAL VALIDATION OF MECHANICS-BASED PROCESS MODELS FOR DIRECT WAFER BONDING
}

\author{
Kevin T. Turner and S. Mark Spearing \\ Massachusetts Institute of Technology \\ Cambridge, MA 02139
}

\begin{abstract}
Direct wafer bonding, also known as fusion bonding, is a common technique used in the fabrication of microsystems. While the process adds tremendous design flexibility, establishing robust processes has often proved to be a challenge. A mechanics-based model to predict failure in direct wafer bonding processes is reported in this work. An energy-based bonding criterion was implemented in a finite element model to allow the shape and size of the bonded area to be predicted as a function of the wafer geometry, etch patterns, elastic properties, and surface chemistry. The modeling approach has been validated through experiments in which the bonded area was predicted across a series of bonded wafers with specific etch patterns and wafer-scale flatness variations. The modeling approach and experiments reported establish a basis for developing advanced mechanics-based process models for wafer bonding.
\end{abstract}

\section{INTRODUCTION}

Direct wafer bonding is an important technology in the manufacture of microsystems. It significantly increases design flexibility, permits the construction of three-dimensional devices, and allows for the integration of dissimilar materials. Direct bonding is not a new technology, originally developed for the fabrication of silicon-on-insulator substrates, the technology has been widely adopted by researchers developing microelectromechanical systems. A range of devices have been realized using direct bonding, including pressure sensors [1], accelerometers, and microfluidic systems [2]. While the technology offers significant benefits and has been demonstrated in numerous devices, process failures have increased as the technique has been employed in the fabrication of more complex devices. In particular, the bonding of multiple wafers has presented significant challenges, as high yields are required in each step and the process is fundamentally more difficult because of the thicker and hence stiffer pieces that must be bonded [3]. The process is further complicated by the need to bond wafers with a high fraction of etched area, which reduces the surface energy available to drive the process, or deposited residually stressed films on the surface, which may increase the surface roughness and deform the wafer. These various factors combine to significantly reduce the process window and make process development considerably more difficult.

Challenges such as these have been acknowledged and empirical solutions developed for specific devices [3]. However, solutions such as these are often time consuming, expensive to develop, and do not readily translate to processes for other devices. As such, there is a clear need to have a fundamental understanding of the direct bonding process and how factors such as etch patterns, wafer geometry (thickness, bow, warp, surface waviness), surface energy, and bonding tools effect process yield. A quantitative understanding of such factors would permit models to be developed that would enable intelligent device and process design and allow tolerances to be set such that pre-bond metrology can be employed. The current work reports a numerical model and (a)

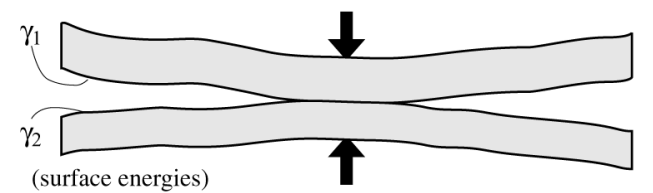

(b)

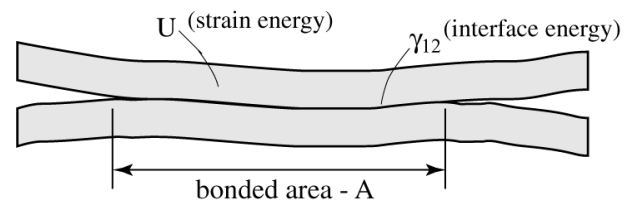

Figure 1. Schematic of wafer deformation during direct bonding.

experiments that demonstrates the effects of wafer geometry and etch patterns may be quantified using a mechanics-based approach.

\section{THEORY AND MODELING}

The basics of the direct bonding process have been understood for some time [4]. In the process, two flat and smooth wafers are initially contacted at room temperature, bond via the formation of short-range van der Waals and hydrogen bonds, and are then subsequently annealed to strengthen the interface. As it is undesirable to apply pressure during the thermal treatment step, it is critical that the wafer surfaces are held in intimate contact by the room temperature bond. Success in the room temperature bond step is contingent on the fact that the surface forces are sufficient to bring the wafers into contact. Wafers are typically not flat (surface waviness, bow, and warp) and as such they must elastically deform to a common shape (Fig. 1) during the bonding process. This fact was recognized early and researchers developed simple expressions that compare the total strain energy required to form a bond to the surface energy available to do so [4,5]. While these expressions demonstrate the essential scaling of bonding difficulty with the geometry and elastic properties of the wafers, they do not provide a clear route to quantitatively connect these factors to bonding failure. These simple expressions proved sufficient for almost a decade, as the majority of bonding processes being developed typically only required the bonding of two prime grade silicon wafers, a relatively easy task that is typically not limited by flatness variations. As more complicated bonding processes have been attempted though [3], it has become clear that a better understanding of the mechanics of the process is required.

A quantitative modeling framework that allows wafer geometry, etch patterns, and surface energy to be related directly to size of the bonded area was reported by current authors in [6]. This modeling framework is based on energy considerations as well, but rather than simply considering the total strain energy as was done in [4], the strain energy per unit area required to advance the bond front was considered. This approach is essentially the application of fracture mechanics to an adhesion problem and is similar to analyses that have been employed in the assessment of stiction in surface micromachined structures [i.e. 7]. As described in [6], the criterion for bond advance is,

$$
\frac{d U}{d A} \leq W
$$

Travel support has been generously provided by the Transducers Research Foundation and by the DARPA MEMS and DARPA BioFlips programs. 

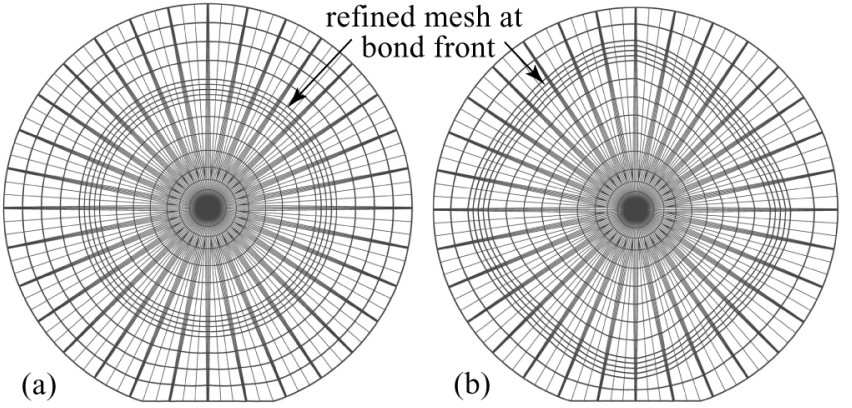

Figure 2. Top view of finite element mesh before (a) and after (b) iteration to determine the equilibrium bond front position.

where $U$ is the strain energy, $A$ is the bond area, and $W$ is the work of adhesion $\left(W=\gamma_{1}+\gamma_{2}-\gamma_{12}\right)$. The term $d U / d A$, the strain energy accumulation rate, is a function of the geometry, elastic properties, and mounting of the wafers and represents the strain energy accumulated in the wafers as the bond advances. In previous work, the case of bonding wafers with axisymmetric curvature (wafer bow) was examined and $d U / d A$ was calculated analytically $[6,8]$. While that work demonstrated the impact that wafer-scale shape variations, etch patterns, and mounting can have on bonding, only qualitative agreement between the analytical model and experiments was achieved [9].

The primary factor that resulted in the differences between the model and experiments in [9] was that the model was axisymmetric, while the wafers in the experiments were asymmetric. In order to obtain better agreement between the model and experiments and to permit the modeling framework proposed in [6] to be applied to real cases with arbitrary geometries and etch patterns, a finite element implementation of Eq. (1) has been developed and is reported here.

The chief difficulty with predicting bonded areas in wafer pairs with asymmetric shape and etch patterns is that the size and shape of the bond front are not known a priori (in the axisymmetric case, the shape of front is known to be circular). The bond area shape and size is determined by the requirement that equilibrium is satisfied along the entire bond front. This requires that $d U / d A=W$ at all points on the bond front. To implement this criterion for a wafer with an arbitrary shape and etch pattern a three dimensional finite element model that allows for $d U / d A$ to be calculated locally along the bond front and for the equilibrium bond shape and size to be determined iteratively was developed.

The bonding of wafers with asymmetric shape and an axisymmetric spoke etch pattern (Fig. 5(b)) was considered. A three-dimensional finite element model with the mesh shown in Fig. 2 was constructed. The wafers have a $100 \mathrm{~mm}$ diameter, a $32.5 \mathrm{~mm}$ long flat along the [110] direction, and the anisotropic elastic properties of silicon. The spoke pattern was defined in the mesh and consisted of a central un-etched area with a $7 \mathrm{~mm}$ radius and 32 spokes that narrow in width from approximately $1.37 \mathrm{~mm}$ at $r=7 \mathrm{~mm}$ to $0.41 \mathrm{~mm}$ at $r=50 \mathrm{~mm}$. The mesh around the bond front was refined. The shape and thickness of the two wafers in the pair were defined from measurements on actual wafers. The model consisted of two elements through the thickness of each wafer and the in-plane distribution shown in Fig. 2. All elements were 20 node solid elements and the mesh density was selected after extensive convergence studies. The mesh was created using a MATLAB script and was solved using the commercial finite element package ABAQUS.

The strain energy accumulation rate, $d U / d A$, was calculated locally along the bond front by adapting the virtual crack closure technique (VCCT) that is commonly used in fracture mechanics to compute the strain energy release rate [10]. Using the VCCT, $d U / d A$ may be calculated along the bond front from the reaction forces, $F$, and the gap between the wafer surfaces, $\delta$, at the nodes of the elements immediately behind and ahead of the bond front,

$$
\frac{d U}{d A}=\frac{1}{2} \frac{F_{i} \delta_{i}}{\Delta A}
$$

where $\Delta \mathrm{A}$ is an area that depends on the element size. Equation (2) is the basic formulation, the exact reaction forces and gaps that are included in the calculation depend on the position of the node (mid-side or corner) and the element type. A comprehensive list of VCCT equations for standard elements may be found in [10]. The expressions in [10] for the VCCT have been developed for fracture analyses. The only change that must be made when applying these equations to an adhesion analysis is in the calculation of the gap ahead of the bond front. For fracture problems, $\delta=u_{A}$ - $u_{B}$, where $u_{A}$ and $u_{B}$ are the nodal displacements of wafer $\mathrm{A}$ and $\mathrm{B}$. In adhesion analyses, there is an initial gap between the nodes at the interface, $\delta_{o}$, that must be accounted for, $\delta=\delta_{o}+u_{A}-u_{B}$. The VCCT was chosen as it allows $d U / d A$ to be calculated locally along a given bond front from a single finite element solution without significant mesh refinement.

The position of the nodes along bond front must be varied in a systematic fashion to determine the equilibrium size and shape. This was accomplished using a quasi-Netwon iteration scheme that was based on work in [11] for predicting crack growth in composites. In this approach, an initial bond shape is assumed, $d U / d A$ is calculated locally along the bond front using the VCCT, and the equilibrium convergence criterion $d U / d A=W+/-1 \%$ is checked. If the front is not in equilibrium, a new bond front position is calculated and $d U / d A$ is evaluated along the new front. This procedure is repeated until convergence is achieved typically 20-50 iterations are required.

Figures 2, 3, and 4 show an example of this modeling process for a real wafer pair. Figure 2(a) shows the initial mesh with an assumed circular bond front. Figure 3 shows maps of the shapes of the two wafers used in this example. One wafer has a large and nearly axisymmetric curvature due to the presence of a residually stressed film while the other has an asymmetric shape variation that is a result of the wafer manufacturing process. Using the model, the shape of front is iterated from the initial assumed shape, Fig. 2(a), to the equilibrium shape, Fig. 2(b). By running the model multiple times for different work of adhesions, the bonded area may be mapped out as a function of $W$. Figure 4 shows the predicted positions of the bond front for several different values of work of adhesion for the wafer pair shown in Fig. 3.

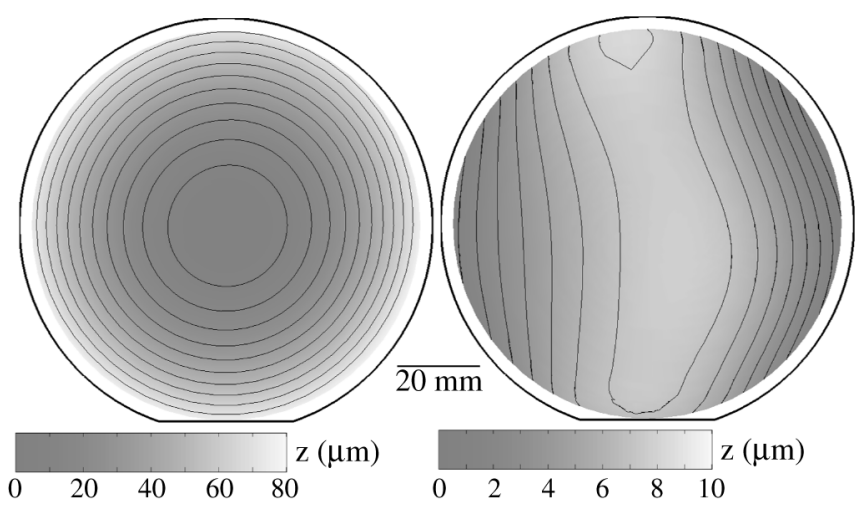

Figure 3. An example shape measurements of two $100 \mathrm{~mm}$ silicon wafers. The 'shape' is defined as the distance between (out-ofplane) the wafer and a reference plane. 


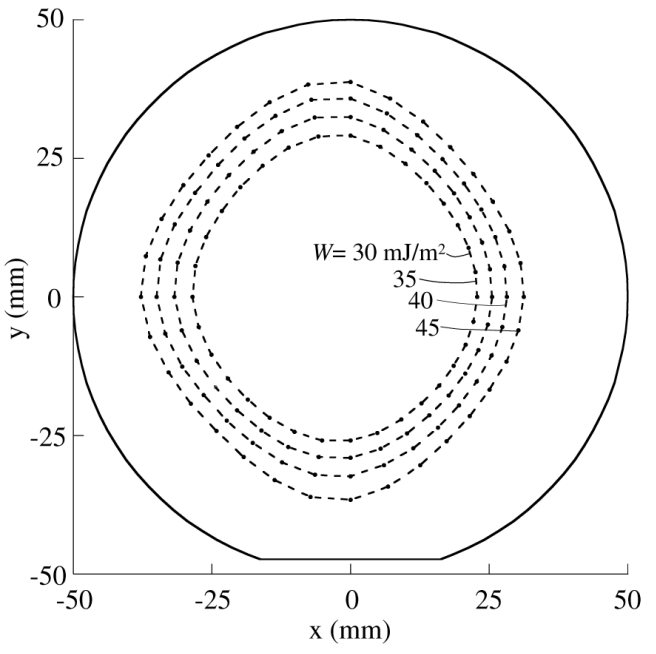

Figure 4. Predicted bond fronts for the wafer pair shown in Fig. 3 for work of adhesion values from $30-45 \mathrm{~mJ} / \mathrm{m}^{2}$.

\section{EXPERIMENTAL DETAILS}

Experiments were conducted in which two $525 \mu \mathrm{m}$ thick, 100 $\mathrm{mm}$, (100) silicon wafers were bonded. The basic experimental configuration, which consisted of one nominally flat wafer with a shallow etch pattern bonded to a wafer with a tensile stressed silicon nitride film on the back surface, is shown in Fig. 5(a). The thickness of the nitride film was varied to obtain different levels of wafer shape. The pattern shown in Fig. 5(b) was etched 2-3 $\mu \mathrm{m}$ deep into one wafer in the pair using deep reactive ion etching. The wafers were cleaned immediately prior to bonding using a standard RCA clean to yield a hydrophilic surface.

The wafers were bonded at room temperature by supporting the wafers at the center and initiating contact with a small pressure. The resulting bond was imaged using a standard IR transmission inspection system [2]. The geometry of each wafer was measured on a commercially available capacitance gage (ADE 9900, ADE Corp., Westwood, MA) that provided maps of the thickness and shape (i.e. Fig. 3) that could be imported directly into the numerical model described. A total of five pairs with a range of different bows (minimum bow $\sim 30 \mu \mathrm{m}$, maximum bow $\sim 80 \mu \mathrm{m})$ were bonded.

\section{RESULTS AND DISCUSSION}

There are two comparisons that may be done to assess the accuracy of the bonding criterion proposed in [6] and the numerical model described in this work: 1) comparison of the predicted shape of the bonded areas to those observed experimentally, and 2) comparison of the work of adhesion values across the different pairs. Since the pairs have different geometries, but similar surface conditions, the extracted work of adhesion values will only be constant among the tests if the model

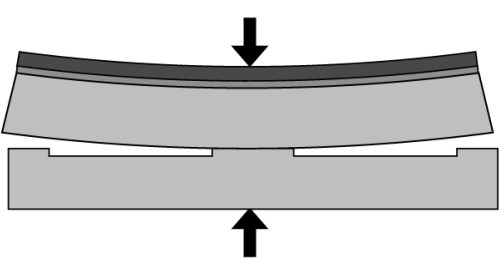

(a)

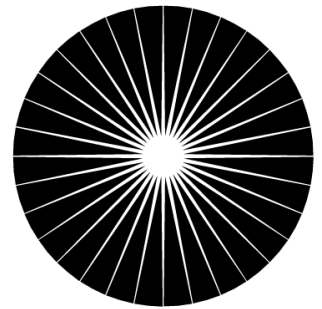

(b)
Figure 5. Bonding configuration (a) and etch pattern (b) used. properly accounts for the geometry of the pairs. It is important to note though that since only wafer scale geometry effects are being measured and accounted for in this work, the work of adhesion value that is being calculated is a function of the surface chemistry as well as smaller scale flatness variations such as roughness and waviness. To ensure that roughness and waviness were consistent across the wafers in the experiments, all wafers were taken from the same manufacturing lot and the roughness (AFM) and waviness (optical profilometry) were measured on several wafers at several locations to ensure consistency.

Of the five pairs that were bonded in this work, one bonded completely and the four others bonded over a portion of the wafer. IR images of the four pairs that bonded partially and bond fronts predicted using the model for each pair are shown in Fig. 6. For each wafer pair, the shapes of the individual wafers were imported into the model and bond fronts were mapped out for a range of work of adhesion values (similar to Fig. 4). The work of adhesion value for each pair was determined by selecting the prediction that matched the experimentally observed front the best. In all of the images in Fig. 6, the prediction with the smaller size corresponds
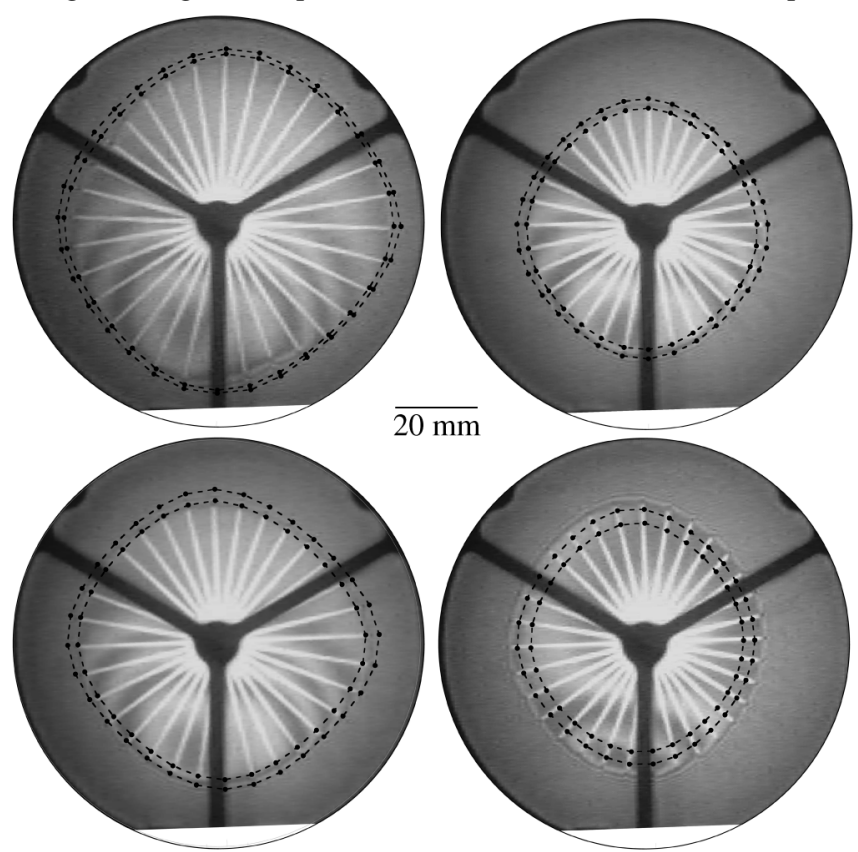

Figure 6. IR transmission images of four bonded wafer pairs and model predictions of the bond front (dashed lines). In all images the predictions correspond to $W=35 \mathrm{~mJ} / \mathrm{m}^{2}$ and $W=40 \mathrm{~mJ} / \mathrm{m}^{2}$.

to a work of adhesion of $35 \mathrm{~mJ} / \mathrm{m}^{2}$ and the larger to $40 \mathrm{~mJ} / \mathrm{m}^{2}$. The work of adhesion values determined from the size of the bonded area and the wafer geometry for each pair using the numerical model reported here and the axisymmetric analytical model reported in [6] are shown in Fig. 7. The large error bars in the axisymmetric calculations are a result of asymmetries in the wafer and bond shape that make defining a single value for the bond front radius and wafer curvature impossible. For the analytical case, the markers in the plot correspond to $W$ if the average values of curvature and bond front position are used in the calculation while the error bars represent the calculated $W$ when the minimum and maximum values of bond radius and curvature are used.

The results in Fig. 6 show that there is good agreement between the shape of the bond front predicted by the model and that observed in experiments. This suggests that the numerical model can accurately account for the effects of wafer shape and etch patterns in bonding. The consistency of the work of adhesion 


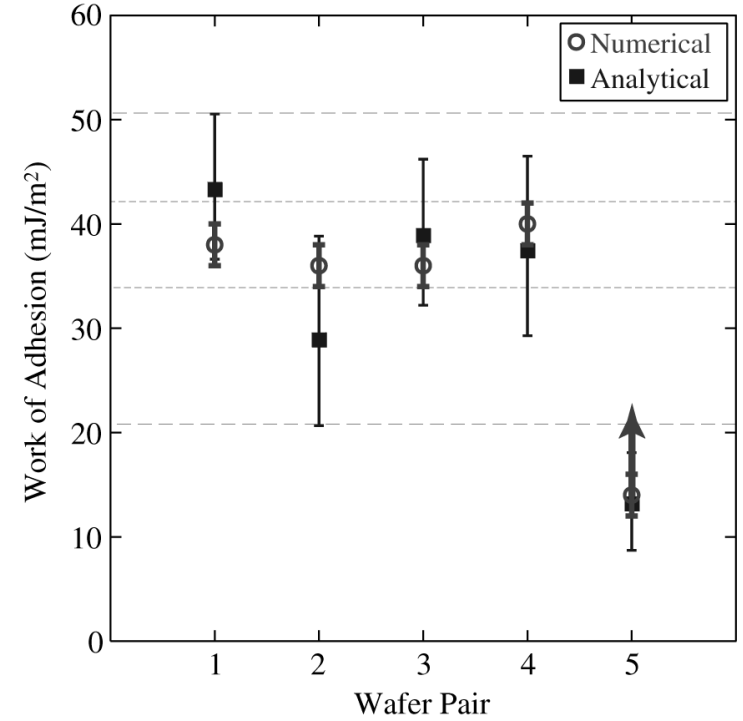

Figure 7. Summary of the work of adhesion values obtained across 5 wafer pairs using the analytical and numerical model.

values predicted across the pairs using the numerical model, as seen in Fig. 6 and 7, further validates the modeling approach and demonstrates that the bonding criterion proposed in [6] is effective in accounting for the effects of wafer geometry and etch patterns in direct wafer bonding. Pair 5 in Fig. 7, is the pair that bonded completely and as such there is no bond front that can be measured to allow for the work of adhesion to be calculated. However, a lower bound on the work of adhesion for that pair may be determined by calculating the work of adhesion required for the bond front to advance to the wafer edge, this is the value plotted in Fig. 7 for pair 5 .

The work of adhesion values calculated from these pairs are reasonable for hydrophilic bonding. The work of adhesion, as used in this context, is a lumped parameter that includes the effects of surface chemistry (types and density of bonds on the surface) and small-scale flatness variations such as roughness. In the experiments performed, the position of the bond front (and hence the work of adhesion) was observed to be a function of time and would grow several millimeters if the pair was left for several hours. To mitigate the effect of time in the experiments all images were taken $10 \mathrm{~min}$ after bonding, the point at which the bond front was observed to not grow a noticeable amount over a 5 minute period. Presumably, the rate at which the bond front grows is associated with the dynamics of bond formation on the surface. To fully understand the nature of the work of adhesion, further studies are clearly required. However, for the purposes of the experiments here, where the primary interest is in the mechanics of the process, comparing work of adhesion values from multiple pairs at a specific time is a good approach.

\section{CONCLUSIONS}

In this work, a numerical model to predict failure in direct wafer bonding processes has been reported. The model incorporates a bonding criterion based on the strain energy accumulated in the wafers during bonding and allows the effect of wafer geometry, etch patterns, elastic properties, and work of adhesion to be accounted for. The model was validated through comparison to experiments, from which it was shown that the size and shape of the bond front is accurately predicted using the model. The experiments described verify the general bonding criterion proposed in [6] and the numerical modeling approach described here.
The results of this work demonstrate that failure in direct wafer bonding processes can be quantified and modeled. The bonding criterion and modeling approach that have been experimentally validated in this work provide a basis for the development of advanced process models that are essential for realizing robust wafer bonding processes.

\section{ACKNOWLEDGEMENTS}

All microfabrication and experiments were performed at the MIT Microsystems Technology Laboratory (MTL). The assistance of the ADE Corporation (P. Hester) in obtaining wafer shape measurements is greatly appreciated. This work was supported by the Cambridge MIT Institute (CMI-059/P-IR(FT) MEMS).

\section{REFERENCES}

1. K. Petersen, P. Barth, J. Poydock, J. Brown, J. Mallon, and J. Bryzek, "Silicon Fusion Bonding for Pressure Sensors," Technical Digest of the 1998 Solid-State Sensor and Actuator Workshop, Hilton Head Isl., SC, 6/6-9/88, IEEE, New York, NY (1988), pp. 144-7.

2. M.A. Schmidt, "Wafer-to-Wafer Bonding for Microstructure Formation," Proc. IEEE, 86, 1575-85 (1998).

3. N. Miki, X. Zhang, R. Khanna, A.A. Ayon, D. Ward, and S.M. Spearing, "A Study of Multi-Stack Silicon-Direct Wafer Bonding for MEMS Manufacturing," Technical Digest MEMS 2002, Las Vegas, NV, 1/20-24/02, IEEE, Piscataway, NJ (2002), pp. 407-10.

4. Q.Y. Tong and U. Goesele, "Semiconductor wafer bonding: recent developments," Mater. Chem. Phys., 37, 101-27 (1994).

5. A. Ploessl and G. Kraeuter, "Wafer direct bonding: tailoring adhesion between brittle materials," Mat. Sci. Eng., R25, 1-88 (1999).

6. K.T. Turner and S.M. Spearing, "Modeling of Direct Wafer Bonding: Effect of Wafer Bow and Etch Patterns," J. Appl. Phys., 92, 7658-66 (2002).

7. M.P. de Boer and T.A. Michalske, "Accurate Method for Determining Adhesion of Cantilever Beams," J. Appl. Phys., 86, 817-27 (1999).

8. K.T. Turner and S.M. Spearing, "Mechanics of Wafer Bonding: Effect of Clamping," J. Appl. Phys., 95, 349-55 (2003).

9. K.T. Turner and S.M. Spearing, "Role of Wafer Bow and Etch Patterns in Direct Bonding," Proceedings of the $7^{\text {th }}$ International Symposium on Semiconductor Wafer Bonding" Science, Technology, and Applications, Paris, France, 4/27-5/2/03, Electrochemical Society, Pennington, NJ (2003), pp. 166-74.

10. R. Krueger, "The Virtual Crack Closure Technique: History, Approach, and Applications," ICASE Report, NASA/CR-2002211628, 1-59 (2002).

11. D. Hitchings, P. Robinson, and F. Javidrad, "A Finite Element Model for Delamination Propagation in Composites," Comput. Struct., 60, 1093-1104 (1996). 